\title{
Local enrichment of finite elements for interface problems
}

\author{
Fausto Cavalli ${ }^{\mathrm{a}}$, Lucia Gastaldi ${ }^{\mathrm{b}, *}$ \\ ${ }^{a}$ Dipartimento di Matematica, Università di Milano, Via Saldini 50, 20133 Milano, Italy. \\ ${ }^{b}$ DICATAM - Sez. Matematica, Università di Brescia, Via Branze 43, 25123 Brescia, \\ Italy.
}

\begin{abstract}
We consider interface problems for second order elliptic partial differential equations with Dirichlet boundary conditions. It is well known that the finite element discretization may fail to produce solutions converging with optimal rates unless the mesh fits with the discontinuity interface. We introduce a method based on piecewise linear finite elements on a non-fitting grid enriched with a local correction on a sub-grid constructed along the interface. We prove that our method recovers the optimal convergence rates both in $H^{1}$ and in $L^{2}$ depending on the local regularity of the solution. Several numerical experiments confirm the theoretical results.

Keywords: Interface problems, finite elements, error estimates
\end{abstract}

\section{Introduction}

In several physical situations, like heat transfer, fluid dynamics, acoustic waves, electromagnetic phenomena and materials science, problems having

\footnotetext{
*Corresponding author. Tel.: +39030 371 5736; Fax:+39 0303715745.

Email addresses: fausto.cavalli@unimi.it (Fausto Cavalli), lucia.gastaldi@ing.unibs.it (Lucia Gastaldi)
} 
discontinuous coefficients across interfaces may arise. In general situations, the position of the interfaces can be either given or can be an unknown of the problem as in the case of phase transition, fluid-structure interaction, heterogeneous structures or free-boundary problems. If the interface is fixed, it might be desirable to construct the mesh in such a way it fits with the interface, in order to achieve the optimal accuracy in each subregion of the domain. In the case of unknown interface as those quoted above, a lot of effort has been made to develop efficient numerical techniques (see for example $[1,2,3,4,5,6,7,8,9,10]$ and the references therein). Since, in this kind of problems, the interface is time dependent and the problem is nonlinear, computing the correct position of the interface at each time step could require some iterations to guarantee stability of the scheme. In such case, instead of adapting the mesh to fit the interface at each time step, it could be convenient considering meshes which do not take into account the position of the interface and apply the enrichment method, we are going to present, to recover the optimal accuracy.

From the mathematical viewpoint, the lack of global regularity of the coefficients may affect the global regularity of the solution, even if the interface is smooth. In these situations numerical schemes may fail to provide the optimal convergence rate. On the other hand, if the coefficients are locally regular, then the solution could enjoy local smoothness properties. Therefore, in order to have a finite element solution which converges to the continuous one with an optimal rate, the computational meshes need to be constructed in such a way that the interface is well approximated by the mesh faces. In this case, the meshes are called fitting. We refer, for example, to $[11,12,13,14]$ for 
the analysis in the elliptic and parabolic case. However, the generation of a fitting grid could be time consuming in presence of complicated geometries or in the case of time dependent interfaces, which would require the re-meshing at each time step. We mention here that one could proceed by constructing independent meshes in the subregions. In such case one gets fitting meshes which could be non-matching, that is grids that do not share the same nodes along the interface between two adjacent regions. For example, one can use a fine mesh on a certain region and a coarser mesh on the adjacent one. As a consequence the finite element space in the first region presents more degrees of freedom along the interface than that defined in the second region. In this situation, modern software packages offer a so-called glue technology which allows to interpolate the two fields in order to obtain a continuous solution, see e.g. ADINA (http://www.adina.com/newsgB36.shtml).

Several techniques have been proposed which employ non-fitting meshes associated with proper strategies which allow to recover the optimal convergence rate. The immersed interface methods proposed in [15, 16] combine the advantages of cartesian grids with the construction of suitable local piecewise polynomials which can take into account the jump of the normal derivative. The partition of unity method [17] allows to include in the finite element spaces a priori knowledge of the behavior of the solution close to the interface. In the extended finite element method [18] an enrichment of the standard finite element spaces is constructed in order to model arbitrary discontinuities of functions or their derivatives. A different approach is followed in the unfitting finite element method proposed in [19] where the approximate solution is allowed to be discontinuous and the correct interface 
conditions are enforced weakly using Nitsche's method. Moreover, we quote the methods based on fictitious domains, see [20, 21].

Our approach is based on the immersed boundary method proposed in [22, 23] for simulating fluid motion around solid objects. In these papers the immersed boundary represents the interface between fluid and solid where Dirichlet boundary conditions are imposed. The main idea of the method is to construct a mesh for the union of the fluid and the solid domains independently of the position of the solid and then to cut the elements crossed by the immersed boundary adding new degrees of freedom. Then the additional unknowns in the solid domain can be computed locally in terms of the new degrees of freedom using the boundary condition. In our case, instead, the immersed boundary corresponds to the interface where the coefficients present discontinuities, therefore the value of the solution along it is not known in advance. In order to obtain a numerical solution with optimal convergence rate, we introduce first a finite element discretization of the interface problem on a non-fitting grid, then we enrich the resulting finite element space performing a local refinement of the grid along the interface. We observe that the additional unknowns we have introduced could be expressed by static condensation in term of the unknowns on the non-fitting grid, so that the size of the algebraic system would not increase. Moreover, this procedure does not require the modification of the non-fitting mesh structure keeping the original numbering of the degrees of freedom. This advantage becomes more relevant when it is applied to the case of time dependent problems with moving interfaces, as, for example, the matrices of the resulting linear system can be modified only locally at each time step. The construction of the sub- 
grid associated with the local enrichment can be done in several ways. Our local finite element space is obtained by subdividing the elements crossed by the interface into sub-elements so that edges can intersect the interface only at the vertexes.

Here we present and discuss into details the case of two dimensional problems discretized by piecewise linear finite elements, however the method can be generalized to higher order finite elements. We show that the method recovers the optimal rate of convergence provided the solution is sufficiently smooth in each subregion. The three dimensional case can also be treated using, for example, the geometric considerations of [22] for the construction of the sub-grid.

The paper is organized as follows: in Sect. 2 we present the problem with the regularity results for the solution; next, the enriched finite element method is introduced and the error estimates are provided in Sect. 4. The last section reports some numerical experiments confirming the theoretical results together with the numerical behavior of the condition number of the involved matrices. In particular, we exploit numerically the behavior of the condition number of the matrix analyzing what happens when the interface gets closer to the existing non-fitting grid so that the sub-elements could fail to satisfy the minimum angle condition.

\section{Elliptic problem with discontinuous coefficients}

In order to simplify the presentation, we consider a convex polygonal domain $\Omega \subset \mathbb{R}^{2}$, divided into two open sets $\Omega_{1}, \Omega_{2}$ by an interface $\Gamma$. The technique we are going to present can be extended to the case of several do- 
mains with suitable modifications. We assume that $\Gamma$ is Lipschitz continuous and that it is composed by a finite number of open arcs each of them of class $\mathcal{C}^{2}$. For a regular function $v$ defined on $\Omega$, we denote by $v_{i}$ for $i=1,2$ its restrictions to $\Omega_{i}$, that is $v_{i}=\left.v\right|_{\Omega_{i}}$ for $i=1,2$.

We consider the following elliptic problem with homogeneous Dirichlet boundary conditions

$$
\begin{array}{ll}
-\nabla \cdot(\beta \nabla u)=f & \text { in } \Omega \\
u=0 & \text { on } \partial \Omega,
\end{array}
$$

where $f$ and $\beta$ are sufficiently smooth functions on $\Omega$. We assume that $\beta \geq \beta_{0}>0$ for some $\beta_{0} \in \mathbb{R}$. The case of non homogeneous Dirichlet boundary conditions can be treated as well with standard modifications.

When $\beta$ presents jump discontinuities across $\Gamma$, problem (1) can be rewritten as the following transmission problem:

$$
\begin{array}{ll}
-\nabla \cdot\left(\beta \nabla u_{1}\right)=f & \text { in } \Omega_{1} \\
-\nabla \cdot\left(\beta \nabla u_{2}\right)=f & \text { in } \Omega_{2} \\
u_{1}=u_{2} & \text { on } \Gamma \\
\beta_{\mid \Omega_{1}} \frac{\partial u_{1}}{\partial \boldsymbol{n}_{1}}+\beta_{\mid \Omega_{2}} \frac{\partial u_{2}}{\partial \boldsymbol{n}_{2}}=0 & \text { on } \Gamma \\
u_{1}=0 & \text { on } \partial \Omega_{1} \backslash \Gamma \\
u_{2}=0 & \text { on } \partial \Omega_{2} \backslash \Gamma .
\end{array}
$$

Let $f \in L^{2}(\Omega)$ and $\beta \in L^{\infty}(\Omega)$. We assume that the restrictions of $\beta$ to the sub-domains $\Omega_{i}$ are sufficiently smooth, that is $\beta_{\mid \Omega_{i}} \in W^{1, \infty}\left(\Omega_{i}\right)$ for $i=1,2$. The variational formulation of problem (1) is then the following: find $u \in H_{0}^{1}(\Omega)$ such that

$$
a(u, v)=(f, v) \quad \forall v \in H_{0}^{1}(\Omega)
$$


where

$$
a(u, v)=\int_{\Omega} \beta \nabla u \nabla v d \boldsymbol{x} \quad(f, v)=\int_{\Omega} f v d \boldsymbol{x} .
$$

We shall use the following notation for the norms in the Hilbert spaces on $B \subset \mathbb{R}^{2}$ : for all $v \in L^{2}(B)$ we set

$$
\|v\|_{0, B}=\left(\int_{B} v^{2} d \boldsymbol{x}\right)^{1 / 2} .
$$

Next for functions $v$ in the Hilbert space $H^{s}(B)$ with $s$ integer we define

$$
\begin{aligned}
& |v|_{j, B}=\left\|D^{j} v\right\|_{0, B} \quad \text { for } 0 \leq j \leq s \\
& \|v\|_{s, B}=\left(\sum_{j=0}^{s}|v|_{j, B}^{2}\right)^{1 / 2}
\end{aligned}
$$

where $D^{j} v$ stands for the array of the derivatives of $v$ of order $j$. When $B=\Omega$ and no confusion may arise we drop the subscript $\Omega$.

We introduce the space $H^{s}\left(\Omega_{1} \cup \Omega_{2}\right)=\left\{v \in H^{1}(\Omega): v_{i} \in H^{s}\left(\Omega_{i}\right)\right.$ for $i=$ $1,2\}$ endowed with the norm

$$
\|v\|_{s, \Omega_{1} \cup \Omega_{2}}=\left(\left\|v_{1}\right\|_{s, \Omega_{1}}^{2}+\left\|v_{2}\right\|_{s, \Omega_{2}}^{2}\right)^{1 / 2}
$$

Problem (3) has a unique solution $u \in H_{0}^{1}(\Omega)$ such that $u \in \cap H^{2}\left(\Omega_{1} \cup \Omega_{2}\right)$ if $\Gamma$ belongs to $C^{2}$, with the following a priori estimate (see $[14,24,25]$ )

$$
\|u\|_{1}+\|u\|_{2, \Omega_{1} \cup \Omega_{2}} \leq C\|f\|_{0} .
$$

In the case when $\Gamma$ does not belong to $C^{2}$ but it is only globally Lipschitz continuous, the regularity of the restrictions of $u$ to $\Omega_{1}$ and $\Omega_{2}$ might be reduced and $u \in H^{s}\left(\Omega_{1} \cup \Omega_{2}\right)$ with $3 / 2<s \leq 2$, see [26, Ch. 2]. 
To simplify the exposition of the numerical method, we assume that the coefficient $\beta$ is piecewise constant with

$$
\beta(x)=\beta_{1} \text { for } x \in \Omega_{1}, \quad \beta(x)=\beta_{2} \text { for } x \in \Omega_{2},
$$

and $\beta_{1}<\beta_{2}$. With this choice it is easy to check that the continuity constant of $a(\cdot, \cdot)$ is given by $\beta_{2}$, and the coercivity constant by $\alpha=2 \beta_{1} /\left(1+C_{\Omega}\right)$ where $C_{\Omega}$ is the constant of the Poincaré inequality.

In the general case, one can obtain the same results by assuming that the restrictions of $\beta$ to the two sub-domains belong to $W^{1, \infty}$, so that the solution achieves the same regularity as in the case of constant coefficients.

\section{Enriched finite element approximation}

In this section we present our method which combines the finite element discretization on a global non-fitting mesh with a local correction obtained by subdividing the elements crossed by the interface into sub-elements matching with it.

Let us consider a regular triangulation $\mathcal{T}_{h}^{G}$ of $\Omega$ with $h=\max _{K \in \mathcal{T}_{h}^{G}} \operatorname{diam}(\mathrm{K})$ and let us introduce the space

$$
V_{h}^{G}=\left\{v_{h}^{G} \in H_{0}^{1}(\Omega): v_{h \mid K}^{G} \in P_{1}(K) \forall K \in \mathcal{T}_{h}^{G}\right\}
$$

where $P_{1}(K)$ denotes the space of affine polynomials on $K$.

For $h$ sufficiently small, $\Gamma$ intersects a generic triangle $K \in \mathcal{T}_{h}^{G}$ in two points, each one on a distinct edge. Notice that the two intersection points might be vertexes of the triangle. We denote by $P_{k}$ for $k=1, \ldots, N_{p}$ the points of intersection of $\Gamma$ with the edges of the elements in $\mathcal{T}_{h}^{G}$ and the points 


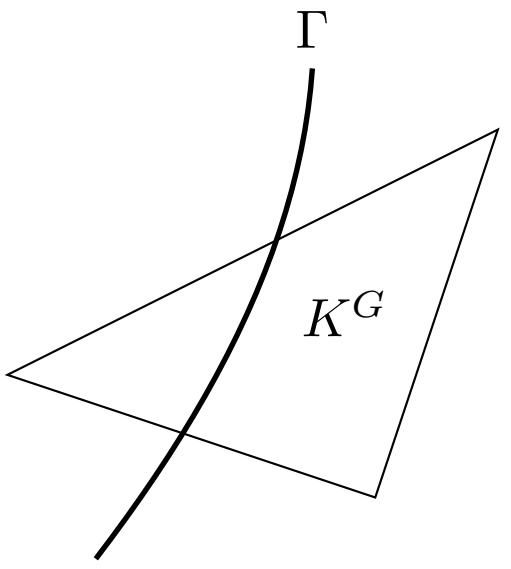

(a) Interface triangle $K^{G} \in \mathcal{T}_{h}^{G}$

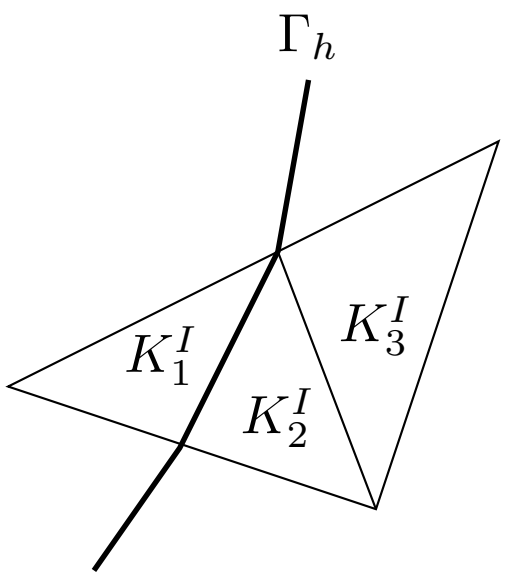

(b) Elements $K^{I} \in \mathcal{T}_{h}^{I}$

Figure 1: An example of a possible interface mesh generation: an interface triangle $K^{G} \in$ $\mathcal{T}_{h}^{G}$ on the left, elements in $\mathcal{T}_{h}^{I}$ obtained by subdivision of $K^{G} \in \mathcal{T}_{h}^{G}$ on the right.

where $\Gamma$ is not regular. Then we connect all these points by a piecewise linear curve $\Gamma_{h}$ which gives an approximation of $\Gamma$. As a consequence $\Omega$ is split into two parts by $\Gamma_{h}$ denoted, with obvious notation, $\Omega_{1, h}$ and $\Omega_{2, h}$. Notice that the triangles which intersect $\Gamma$ are not contained either in $\Omega_{1, h}$ or in $\Omega_{2, h}$ so that $\Omega_{i, h}$ for $i=1,2$ are not the union of elements in $\mathcal{T}_{h}^{G}$. Let us introduce the following approximation of the coefficient $\beta$

$$
\beta_{h}(x)=\beta_{1} \quad \text { for } x \in \Omega_{1, h} \quad \beta_{h}(x)=\beta_{2} \quad \text { for } x \in \Omega_{2, h} .
$$

Let $\Omega^{I}$ be the union of the interface elements of the triangulation $\mathcal{T}_{h}^{G}$ that is

$$
\Omega^{I}=\bigcup_{\substack{K \in \mathcal{T}^{G} \\ K \cap \Gamma \neq \emptyset}} K .
$$

Then also $\Omega^{I}$ is split by $\Gamma_{h}$ into two parts $\Omega_{1, h}^{I} \subset \Omega_{1, h}$ and $\Omega_{2, h}^{I} \subset \Omega_{2, h}$. Now we construct a triangulation $\mathcal{T}_{h}^{I}$ of $\Omega^{I}$ such that for all $K^{I} \in \mathcal{T}_{h}^{I}$ we have that 
$\Gamma_{h} \cap K^{I}$ is either an edge or a vertex and $K^{I} \subseteq K^{G}$ for some $K^{G} \in \mathcal{T}_{h}^{G}$, see Fig. 1. Since each element $K^{I} \in \mathcal{T}_{h}^{I}$ is contained either in $\Omega_{1, h}$ or in $\Omega_{2, h}, \beta_{h}$ is constant in each $K^{I}$. Moreover we have the following characterization for $i=1,2$

$$
\Omega_{i, h}=\left(\bigcup_{\substack{K^{G} \in \mathcal{T}_{h}^{G}, K^{G} \subset \Omega_{i}}} K^{G}\right) \bigcup\left(\bigcup_{\substack{K^{I} \in \mathcal{T}_{h}^{I}, K^{I} \subset \Omega_{i, h}^{I}}} K^{I}\right) .
$$

We assume that the mesh $\mathcal{T}_{h}^{I}$ is also regular, that is there exists $\delta_{I}$ such that for all $K \in \mathcal{T}_{h}^{I}$ it holds

$$
\rho_{K} \geq \delta_{I} h_{K}
$$

where $\rho_{K}$ and $h_{K}$ stand for the radius of the largest ball contained in $K$ and the diameter of $K$, respectively.

In order to respect the regularity assumption on the mesh, one should avoid anisotropic elements and this can be achieved using suitable different strategies for the construction of the sub-grid.

Let us consider the space

$$
V_{h}^{I}=\left\{v_{h}^{I} \in H_{0}^{1}(\Omega): v_{h \mid K}^{I} \in P_{1}(K) \forall K \in \mathcal{T}_{h}^{I}, v_{h}^{I}=0 \text { in } \Omega \backslash \Omega^{I}\right\},
$$

then the finite element space we are going to use in the discretization of problem (3) is obtained by direct sum of the previous two subspaces

$$
V_{h}=V_{h}^{G}+V_{h}^{I}
$$

Writing any $v_{h} \in V_{h}$ as the sum $v_{h}=v_{h}^{G}+v_{h}^{I}$ with $v_{h}^{G} \in V_{h}^{G}$ and $v_{h}^{I} \in V_{h}^{I}$, we 
define the following bilinear forms:

$$
\begin{array}{ll}
a_{G}\left(u_{h}^{G}, v_{h}^{G}\right)=\sum_{K \in \mathcal{T}_{h}^{G}} \int_{K} \beta_{h} \nabla u_{h}^{G} \nabla v_{h}^{G} d x & \forall u_{h}^{G}, v_{h}^{G} \in V_{h}^{G} \\
a_{I G}\left(u_{h}^{I}, v_{h}^{G}\right)=\sum_{i=1}^{2} \int_{\Omega_{i, h}^{I}} \beta_{h} \nabla u_{h}^{I} \nabla v_{h}^{G} d x & \forall u_{h}^{I} \in V_{h}^{I}, v_{h}^{G} \in V_{h}^{G} \\
a_{G I}\left(u_{h}^{G}, v_{h}^{I}\right)=\sum_{i=1}^{2} \int_{\Omega_{i, h}^{I}} \beta_{h} \nabla u_{h}^{G} \nabla v_{h}^{I} d x & \forall u_{h}^{G} \in V_{h}^{G}, v_{h}^{I} \in V_{h}^{I} \\
a_{I}\left(u_{h}^{I}, v_{h}^{I}\right)=\sum_{K \in \mathcal{T}_{h}^{I}} \int_{K} \beta_{h} \nabla u_{h}^{I} \nabla v_{h}^{I} d x & \forall u_{h}^{I}, v_{h}^{I} \in V_{h}^{I} .
\end{array}
$$

Then the discretized counterpart of (3) reads: find $u_{h} \in V_{h}$ such that

$$
a_{h}\left(u_{h}, v_{h}\right)=\left(f, v_{h}\right) \quad \forall v_{h} \in V_{h},
$$

where for all $u_{h}, v_{h} \in V_{h}$

$$
a_{h}\left(u_{h}, v_{h}\right)=a_{G}\left(u_{h}^{G}, v_{h}^{G}\right)+a_{I G}\left(u_{h}^{I}, v_{h}^{G}\right)+a_{G I}\left(u_{h}^{G}, v_{h}^{I}\right)+a_{I}\left(u_{h}^{I}, v_{h}^{I}\right) .
$$

Due to the construction of the finite element space $V_{h}$, we can write $u_{h}=u_{h}^{G}+u_{h}^{I}$ with $u_{h}^{G} \in V_{h}^{G}$ and $u_{h}^{I} \in V_{h}^{I}$ satisfying the following system:

$$
\begin{array}{ll}
a_{G}\left(u_{h}^{G}, v_{h}^{G}\right)+a_{I G}\left(u_{h}^{I}, v_{h}^{G}\right)=\left(f, v_{h}^{G}\right) & \forall v_{h}^{G} \in V_{h}^{G} \\
a_{G I}\left(u_{h}^{G}, v_{h}^{I}\right)+a_{I}\left(u_{h}^{I}, v_{h}^{I}\right)=\left(f, v_{h}^{I}\right) & \forall v_{h}^{I} \in V_{h}^{I} .
\end{array}
$$

Here, we have considered triangular meshes, but the same technique can be also applied when $\mathcal{T}_{h}^{G}$ is made by quadrilateral elements. Some difficulties arise when the interface cuts a quadrilateral across two adjacent edges. In this case a triangle is generated. This suggests the use of a mixture of triangles and quadrilateral in the fine mesh, or the introduction of a local triangular mesh. We observe that the local correction is totally independent 
from the global finite element space, so that we can subdivide each quadrilateral element crossed by the interface by triangles and use again the space $V_{h}^{I}$ defined in (9). We point out also that the above construction can be generalized to the three-dimensional case. For the construction of the sub-grid we refer to [22] where a way for subdividing the tetrahedral elements crossed by the interface is described.

\section{Error estimates}

In this section we study the convergence analysis of our scheme using the technique developed in $[27,28]$. All along this section we assume that $\Gamma$ is of class $\mathcal{C}^{2}$, so that $u \in H^{2}\left(\Omega_{1} \cup \Omega_{2}\right)$. The general case of a Lipschitz interface will be discussed in Remark 1 at the end of this section.

First of all we prove an a priori estimate for the discrete solution. Assuming that the integrals are computed exactly, it is not difficult to see that the bilinear form $a_{h}$ is uniformly elliptic: for all $v_{h} \in V_{h}$ it holds

$$
a_{h}\left(v_{h}, v_{h}\right) \geq \alpha\left\|v_{h}\right\|_{1}^{2}
$$

where $\alpha=2 \beta_{1} /\left(1+C_{\Omega}\right)$ as in the continuous case.

Since $V_{h} \subset H_{0}^{1}(\Omega)$, we have that problem (10) has a unique solution $u_{h}$ with the following a priori estimate

$$
\left\|u_{h}\right\|_{1} \leq \frac{C_{\Omega}}{\alpha}\|f\|_{0}
$$

With standard arguments, one can prove the following version of the Strang Lemma: 
Lemma 1. Let $u \in H_{0}^{1}(\Omega)$ and $u_{h} \in V_{h}$ be the solutions to (3) and (10) respectively, then

$$
\left\|u-u_{h}\right\|_{1} \leq \frac{1+\beta_{2}}{\alpha} \inf _{v_{h} \in V_{h}}\left\|u-v_{h}\right\|_{1}+\frac{1}{\alpha} \sup _{w_{h} \in V_{h}} \frac{a_{h}\left(u, w_{h}\right)-\left(f, w_{h}\right)}{\left\|w_{h}\right\|_{1}} .
$$

In order to estimate the two terms appearing in the right hand side of (13), we introduce the following notation. For $i=1,2$ we write

$$
\tau_{h}^{i}=\Omega_{i, h} \backslash \Omega_{i}, \quad \omega_{h}^{i}=\Omega_{i} \backslash \Omega_{i, h}
$$

It is easy to verify that $\tau_{h}^{1}=\omega_{h}^{2}$ and vice versa $\tau_{h}^{2}=\omega_{h}^{1}$. For $i=1,2$ let $\tilde{\Omega}^{i}$ be such that $\tilde{\Omega}_{i} \supset \Omega_{i} \cup \Omega_{i, h}$. For any $v_{i} \in H^{2}\left(\Omega_{i}\right)$ then $\tilde{v}_{i} \in H^{2}\left(\tilde{\Omega}_{i}\right)$ denotes the Calderón extension of $v_{i}$ from $H^{2}\left(\Omega_{i}\right)$ into $H^{2}\left(\tilde{\Omega}_{i}\right)$ such that $\tilde{v}_{i}=v_{i}$ in $\Omega_{i}$ and the following estimate holds true (see, e.g. [29])

$$
\left\|\tilde{v}_{i}\right\|_{2, \tilde{\Omega}_{i}} \leq C\left\|v_{i}\right\|_{2, \Omega_{i}}
$$

The following lemma can be proved using the same arguments as in $[28$, Lemmas 3.3.11 and 3.3.12].

Lemma 2. There exists a constant $C$ independent of $h$ such that for $i=1,2$ and $j=1,2$ with $j \neq i$

$$
\|v\|_{0, \tau_{h}^{i}} \leq C h\|v\|_{1, \Omega_{j}} \quad \forall v \in H^{1}(\Omega) .
$$

Moreover, for every $v_{h} \in V_{h}$ let us denote by $v_{i, h}$ its restriction to $\Omega_{i, h}$, then for $i=1,2$ it holds

$$
\begin{aligned}
& \left\|\nabla v_{i, h}\right\|_{0, \tau_{h}^{i}} \leq C h^{1 / 2}\left\|v_{i, h}\right\|_{1, \Omega_{i, h}} \\
& \left\|v_{i, h}\right\|_{0, \tau_{h}^{i}} \leq C h\left\|v_{i, h}\right\|_{1, \Omega_{i, h}}
\end{aligned}
$$

where the constants $C$ do not depend on $h$. 
Following [27] we now prove an interpolation error estimate. Let us define the interpolation operator $\pi_{h}$ for all $v \in C^{0}(\bar{\Omega})$ :

$$
\pi_{h}(v)=\pi_{h, G}(v)+\pi_{h, I}\left(v-\pi_{h, G}(v)\right)
$$

where $\pi_{h, G}(v) \in V_{h}^{G}$ and $\pi_{h, I}(v) \in V_{h}^{I}$ are the interpolation of $v$ in $V_{h}^{G}$ and $V_{h}^{I}$, respectively.

Lemma 3. If $\mathcal{T}_{h}^{G}$ and $\mathcal{T}_{h}^{I}$ are regular triangulations and $v \in C^{0}(\bar{\Omega}) \cap H^{2}\left(\Omega_{1} \cup\right.$ $\left.\Omega_{2}\right)$, then we have

$$
\left|v-\pi_{h}(v)\right|_{1, \Omega} \leq C h\left\|v_{1}\right\|_{2, \Omega_{1} \cup \Omega_{2}},
$$

where $v_{1}$ and $v_{2}$ are the restrictions of $v$ to $\Omega_{1}$ and $\Omega_{2}$, respectively.

Proof. We can write

$$
\left|v-\pi_{h}(v)\right|_{1, \Omega}^{2}=\left|v-\pi_{h}(v)\right|_{1, \Omega \backslash \Omega^{I}}^{2}+\left|v-\pi_{h}(v)\right|_{1, \Omega^{I}}^{2}
$$

Since $\pi_{h, I}(v)$ vanishes on $\Omega \backslash \Omega^{I}$ and $\mathcal{T}_{h}^{G}$ is regular, applying usual interpolation error estimates (see e.g. [30]), we have that

$$
\left|v-\pi_{h}(v)\right|_{1, \Omega \backslash \Omega^{I}}=\left|v-\pi_{h, G}(v)\right|_{1, \Omega \backslash \Omega^{I}} \leq C_{1} h|v|_{2, \Omega \backslash \Omega^{I}} .
$$

It remains to bound the term on the interface domain $\Omega^{I}$. We split it as follows

$$
\left|v-\pi_{h}(v)\right|_{1, \Omega^{I}}^{2}=\sum_{i=1}^{2}\left|v-\pi_{h}(v)\right|_{1, \Omega_{i, h}^{I}}^{2} .
$$

By construction we have that the triangles $K^{I} \in \mathcal{T}_{h}^{I}$ can have at most two vertexes on $\Gamma$. If $K^{I}$ is contained either in $\Omega_{1}$ or $\Omega_{2}$, thanks to (8), we 
can apply standard interpolation error estimates taking into account that $\pi_{h}(v) \in P_{1}\left(K^{I}\right)$

$$
\left|v-\pi_{h}(v)\right|_{1, K^{I}} \leq C h|v|_{2, K^{I}}
$$

Let us now consider elements $K^{I}$ contained in $\Omega_{i, h}^{I}$ for $i=1,2$ with a nonempty intersection with $\Omega_{j}$ where $j=1,2$ and $j \neq i$. Let us remember that the restriction $v_{i} \in H^{2}\left(K^{I} \cap \Omega_{i}\right)$ and that $\tilde{v}_{i}$ denotes its Calderón extension to $\tilde{\Omega}_{i}$. Then we can write

$$
\begin{aligned}
\left|v-\pi_{h}(v)\right|_{1, K^{I}}^{2} & =\int_{K_{I} \cap \Omega_{i}}\left|\nabla\left(v_{i}-\pi_{h}(v)\right)\right|^{2} d x+\int_{K_{I} \cap \Omega_{j}}\left|\nabla\left(v_{j}-\pi_{h}(v)\right)\right|^{2} d x \\
& \leq 2 \int_{K_{I}}\left|\nabla\left(\tilde{v}_{i}-\pi_{h}(v)\right)\right|^{2} d x+2 \int_{K_{I} \cap \Omega_{j}}\left|\nabla\left(v_{j}-\tilde{v}_{i}\right)\right|^{2} d x
\end{aligned}
$$

Summing all these terms we have

$$
\left|v-\pi_{h}(v)\right|_{1, \Omega_{i, h}^{I}}^{2} \leq 2\left(\left|v_{j}-\tilde{v}_{i}\right|_{1, \tau_{h}^{i}}^{2}+\left|\tilde{v}_{i}-\pi_{h}(v)\right|_{1, \Omega_{i, h}^{I}}^{2}\right)
$$

By definition of $\tilde{v}_{i}$ we have that the restriction of $\pi(v)$ to each sub-domain $\Omega_{i, h}^{I}$ coincides with the interpolation of $\tilde{v}_{i}$, hence using standard interpolation estimates and (14) we get

$$
\left|\tilde{v}_{i}-\pi_{h}(v)\right|_{1, \Omega_{i, h}^{I}}^{2} \leq C h^{2}\left\|v_{i}\right\|_{2, \Omega_{i}}^{2}
$$

Moreover, thanks to Lemma 2 and (14), we have

$$
\left|v_{j}-\tilde{v}_{i}\right|_{1, \tau_{h}^{i}}^{2} \leq C h^{2}\left\|v_{j}\right\|_{2, \Omega_{j} \cup \Omega_{i}}^{2}
$$

Putting together (18), (19) and the last three inequalities (20)-(22) we obtain (17).

For the estimate of the second term in (13) we prove the following lemma. 
Lemma 4. Let $u$ be the solution to (3), then we have the following estimate for all $w_{h} \in V_{h}$

$$
a_{h}\left(u, w_{h}\right)-\left(f, w_{h}\right) \leq C h^{3 / 2}\|u\|_{2, \Omega_{1} \cup \Omega_{2}}\left\|w_{h}\right\|_{1},
$$

where $C$ is a constant independent of $h$, but depends on $\beta_{2}$.

Proof. Since $u$ is the solution of problem (3) and $V_{h} \subset H_{0}^{1}(\Omega)$, then

$$
a_{h}\left(u, w_{h}\right)-\left(f, w_{h}\right)=a_{h}\left(u, w_{h}\right)-a\left(u, w_{h}\right) .
$$

The two bilinear forms differ only on the sets $\tau_{h}^{i}$ and $\omega_{h}^{i}$ for $i=1,2$. In fact, recalling the definition (7) of $\beta_{h}$, we have

$$
\begin{aligned}
a_{h}\left(u, w_{h}\right)-a\left(u, w_{h}\right) & =\sum_{i=1}^{2}\left(\int_{\Omega_{i, h}} \beta_{h} \nabla u \nabla w_{h} d x-\int_{\Omega_{i}} \beta \nabla u \nabla w_{h} d x\right) \\
& =\sum_{i=1}^{2} \int_{\tau_{h}^{i}} \beta_{i} \nabla u \nabla w_{h} d x \\
& \leq C \sum_{i=1}^{2} \beta_{i}|u|_{1, \tau_{h}^{i}}\left|w_{h}\right|_{1, \tau_{h}^{i}} .
\end{aligned}
$$

Then, using Lemma 2, we obtain the desired result.

The results of Lemmas 1-4 give the following theorem:

Theorem 1. Let $u \in H_{0}^{1}(\Omega) \cap H^{2}\left(\Omega_{1} \cap \Omega_{2}\right)$ be the solution to problem (3) and $u_{h} \in V_{h}$ be the solution of (10), then the following error estimate holds true

$$
\left|u-u_{h}\right|_{1} \leq C h\|u\|_{2, \Omega_{1} \cup \Omega_{2}}
$$

where $C$ is independent of $h$, but depends on the values of $\beta_{1}$ and $\beta_{2}$.

The $L^{2}$ error estimate can be derived from (23) using a duality argument. 
Theorem 2. Let $u$ and $u_{h}$ be as in Theorem 1, then we have

$$
\left\|u-u_{h}\right\|_{0} \leq C h^{2}\|u\|_{2, \Omega_{1} \cup \Omega_{2}},
$$

with $C$ independent of $h$ but depending on the ratio $\beta_{2} / \beta_{1}$.

Proof. Let us consider the following auxiliary problem: find $\varphi \in H_{0}^{1}(\Omega)$ such that

$$
a(\varphi, v)=\left(u-u_{h}, v\right) \quad \forall v \in H_{0}^{1}(\Omega),
$$

where the bilinear form $a$ is defined in (4). As for the solution of problem (3) we have that $\varphi \in H^{2}\left(\Omega_{1} \cup \Omega_{2}\right)$ with the following a priori estimate

$$
\|\varphi\|_{1}+\|\varphi\|_{2, \Omega_{1} \cup \Omega_{2}} \leq C\left\|u-u_{h}\right\|_{0} .
$$

Taking $v=u-u_{h}$ in (25) we have

$$
\begin{aligned}
\left\|u-u_{h}\right\|_{0}^{2}= & a\left(\varphi, u-u_{h}\right) \\
= & a\left(\varphi, u-u_{h}\right)-a_{h}\left(\varphi, u-u_{h}\right) \\
& +a_{h}\left(\varphi-\pi_{h}(\varphi), u-u_{h}\right)+a_{h}\left(\pi_{h}(\varphi), u-u_{h}\right) .
\end{aligned}
$$

Using Lemmas 2 and 3 and inequality (26), we obtain

$$
\begin{aligned}
a\left(\varphi, u-u_{h}\right) & -a_{h}\left(\varphi, u-u_{h}\right) \\
& =\sum_{i=1}^{2}\left(\int_{\Omega_{i}} \beta \nabla \varphi \nabla\left(u-u_{h}\right) d x-\int_{\Omega_{i, h}} \beta_{h} \nabla \varphi \nabla\left(u-u_{h}\right) d x\right) \\
& =\sum_{i=1}^{2} \int_{\omega_{h}^{i}} \beta_{i} \nabla \varphi \nabla\left(u-u_{h}\right) d x \\
& \leq C \sum_{i=1}^{2}\|\nabla \varphi\|_{0, \omega_{h}^{i}}\left\|\nabla\left(u-u_{h}\right)\right\|_{0, \omega_{h}^{i}} \\
& \leq C h\|\varphi\|_{2, \Omega_{1} \cup \Omega_{2}}\left\|u-u_{h}\right\|_{1} \\
& \leq C h\left\|u-u_{h}\right\|_{0}\left\|u-u_{h}\right\|_{1},
\end{aligned}
$$


and

$$
a_{h}\left(\varphi-\pi_{h}(\varphi), u-u_{h}\right) \leq C\left|\varphi-\pi_{h}(\varphi)\right|_{1}\left|u-u_{h}\right|_{1} \leq C h\left\|u-u_{h}\right\|_{0}\left\|u-u_{h}\right\|_{1} .
$$

For the last term in (27) we have

$$
a_{h}\left(\pi_{h}(\varphi), u-u_{h}\right)=a_{h}\left(u, \pi_{h}(\varphi)\right)-\left(f, \pi_{h}(\varphi)\right)
$$

hence we use the same proof as in Lemma 4 arriving at

$$
\begin{aligned}
a_{h}\left(\pi_{h}(\varphi), u-u_{h}\right) & \leq C \sum_{i=1}^{2}|u|_{1, \tau_{h}^{i}}\left|\pi_{h}(\varphi)\right|_{1, \tau_{h}^{i}} \\
& \leq \sum_{i=1}^{2}|u|_{1, \tau_{h}^{i}}\left(|\varphi|_{1, \tau_{h}^{i}}+\left|\pi_{h}(\varphi)-\varphi\right|_{1, \tau_{h}^{i}}\right) \\
& \leq C h\|u\|_{2, \Omega_{1} \cup \Omega_{2}}\left(C h\|\varphi\|_{2, \Omega_{1} \cup \Omega_{2}}+\left|\pi_{h}(\varphi)-\varphi\right|_{1}\right) \\
& \leq C h^{2}\|u\|_{2, \Omega_{1} \cup \Omega_{2}}\|\varphi\|_{2, \Omega_{1} \cup \Omega_{2}} \\
& \leq C h^{2}\|u\|_{2, \Omega_{1} \cup \Omega_{2}}\left\|u-u_{h}\right\|_{0} .
\end{aligned}
$$

Inserting (28)-(30) in (27) and using (23) we obtain the desired estimate.

Remark 1. Let us assume that the interface $\Gamma$ is only Lipschitz continuous and that it can be subdivided into a finite number of arcs each of them of class $\mathcal{C}^{2}$. In this case we have that $u \in H^{s}\left(\Omega_{1} \cup \Omega_{2}\right)$ with $3 / 2<s \leq 2$, see [26, Ch. 2]. The Calderón extension cannot be applied anymore, since in the case of Lipschitz continuous boundary, it is defined only for functions in $H^{m}$ with integer $m$ [29]. Nevertheless, the results of this section can be extended to the case of Lipschitz interface, provided that we use the general extension operator $\mathcal{E}: \mathcal{C}^{\infty}(\bar{\Omega}) \rightarrow: \mathcal{C}^{\infty}\left(\mathbb{R}^{2}\right)$ introduced by Stein [31, Th.5 pp.181], extended to 
$H^{m}(\Omega)$ with $m \in \mathbb{N}$ by a density argument and generalized to fractional Sobolev spaces by interpolation [32, Th. B.2]. Therefore we have the following error estimates:

$$
\left\|u-u_{h}\right\|_{0}+h\left|u-u_{h}\right|_{1} \leq C h^{s}\|u\|_{s, \Omega_{1} \cup \Omega_{2}} .
$$

Remark 2. The approach we used can be extended to higher order finite elements. We point out that, in such case, we could achieve convergence rates similar to (23) and (24) provided that $u \in H^{s}\left(\Omega_{1} \cup \Omega_{2}\right)$ with $s>2$. We recall that the regularity of the solution depends on the regularity of the interface. For example, if the interface is a straight line, we can obtain

$$
\left\|u-u_{h}\right\|_{m} \leq C h^{k+1-m}\|u\|_{H^{k+1}\left(\Omega_{1} \cup \Omega_{2}\right)}
$$

with $m=0,1$ and $k$ the degree of the finite elements. If this is not the case, it is necessary to introduce higher order approximation of the interface to achieve the desired convergence rate, and this implies the use of isoparametric finite elements. We are not going to investigate further these aspects in this work.

\section{Numerical results}

In this section we provide some details about the implementation of the method introduced in Section 3 and present several numerical results which confirm the analysis presented in Section 4. Moreover, we discuss how to construct and solve the resulting linear system. We investigate also some aspects related to the behavior of the condition number of the matrices involved in the solution of the linear system and we compare our approach with a different technique for the solution of interface problems. 


\subsection{Implementation}

As remarked in section 3 , we write the solution as $u_{h}=u_{h}^{G}+u_{h}^{I}$ where $u_{h}^{G} \in V_{h}^{G}$ and $u_{h}^{I} \in V_{h}^{I}$, in order to obtain system (11). Assuming that

$$
V_{h}^{G}=\operatorname{span}\left\{\phi_{i}^{G} i=1, \ldots n\right\}, \quad V_{h}^{I}=\operatorname{span}\left\{\phi_{i}^{I} i=1, \ldots m\right\}
$$

and writing

$$
u_{h}^{G}=\sum_{j=1}^{n} u_{j}^{G} \phi_{j}^{G}, \quad u_{h}^{I}=\sum_{j=1}^{m} u_{j}^{I} \phi_{j}^{I},
$$

system (11) leads to the algebraic system $A u=b$, with

$$
A=\left(\begin{array}{cc}
A_{G G} & A_{G I} \\
A_{I G} & A_{I I}
\end{array}\right), \quad u=\left(\begin{array}{c}
u_{G} \\
u_{I}
\end{array}\right), \quad b=\left(\begin{array}{c}
b_{G} \\
b_{I}
\end{array}\right),
$$

where the matrix $A_{X Y}$ has elements given by $a\left(\phi_{j}^{Y}, \phi_{i}^{X}\right)$, similarly $u_{X}$ and $b_{X}$ are vectors with components $u_{i}^{X}$ and $\left(f, \phi_{i}^{X}\right)$, respectively. We remark that the diagonal blocks are symmetric and positive definite and that $A_{G I}=A_{I G}^{\top}$, so that system (31) is symmetric, positive definite and sparse. To solve numerically system (31), different approaches can be taken into account. Among others, we can solve directly system $A u=b$ by using any suitable sparse iterative solver for symmetric and positive definite systems or we can consider the block partitioned system (31), compute the Schur complement matrix

$$
A_{S}=A_{G G}-A_{G I} A_{I I}^{-1} A_{I G}
$$

and solve the two smaller systems $A_{S} u_{G}=b_{G}-A_{G I} A_{I I}^{-1} b_{I}$ and $A_{I I} u_{I}=$ $b_{I}-A_{I G} u_{G}$. In the following we give an estimate of the condition number of the matrices $A, A_{S}$ and of the two blocks $A_{G G}$ and $A_{I I}$. 


\subsection{Error analysis}

In all the following simulations, we considered two different families of meshes. The first one is made up of structured meshes, which are obtained by dividing each element of a Cartesian grid into two triangles; the second one is made up of general regular unstructured meshes. Each mesh is obtained by refining increasingly the coarsest mesh of the family. As an example, we report in Figure 2 a mesh for each family.
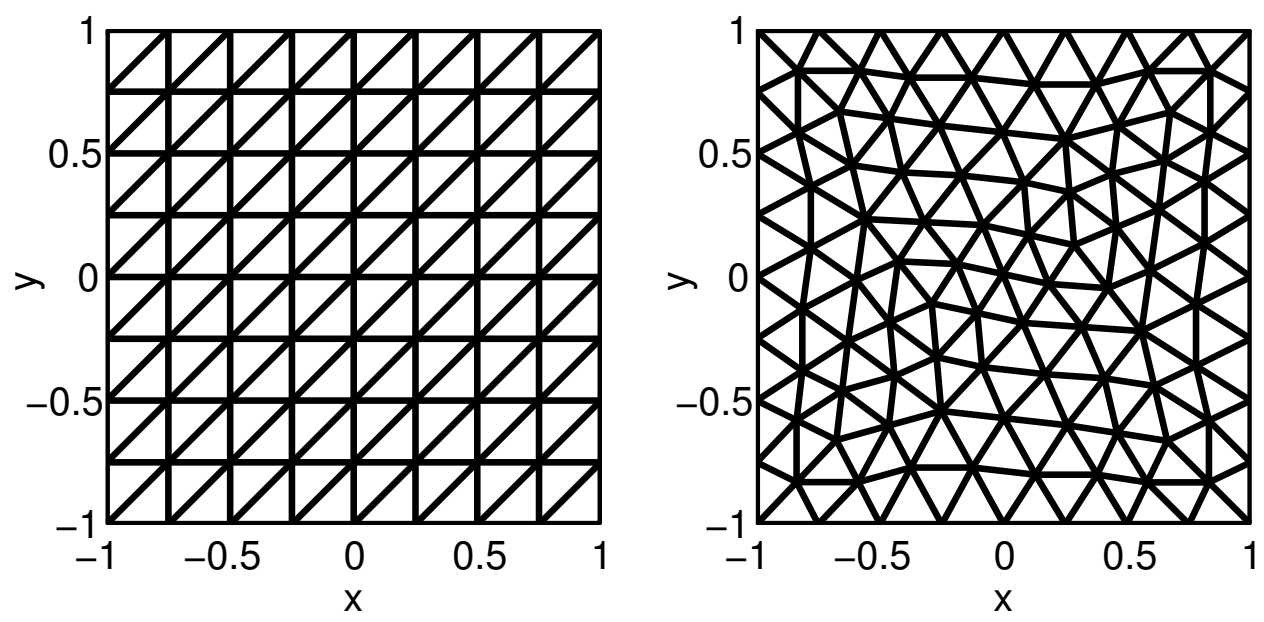

Figure 2: Example of a structured (left) and unstructured (right) meshes.

Let us set $\Omega=[-1,1]^{2}, \Gamma=\{(x, y) \in \Omega: x=\gamma(y)\}$ with $\gamma(y)=c_{1} y^{2}+$ $c_{2} y+c_{3}, \Omega_{1}=\{(x, y) \in \Omega: x<\gamma(y)\}$ and $\Omega_{2}=\{(x, y) \in \Omega: x>\gamma(y)\}$, then we consider the elliptic problem (2) with piecewise constant coefficient given by (6) and $f$ such that the solution is the following piecewise smooth 
function

$$
u(x, y)= \begin{cases}\beta_{2} \sin (2 \pi(x-\gamma(y))) \sin (2 \pi y) & (x, y) \in \Omega_{1}, \\ \beta_{1} \sin (2 \pi(x-\gamma(y))) \sin (2 \pi y) & (x, y) \in \Omega_{2} .\end{cases}
$$

We notice that for all choices of $\beta_{1}, \beta_{2}, c_{i}$ for $i=1,2,3$, the solution given in (33) verifies both transmission conditions in (2).

First we check the convergence of the method for two different couples of constants $\beta_{i}, i=1,2$ considering as interface both a straight line and a curve.

In these simulations we use the family of unstructured meshes, made of $46 \cdot 4^{m}, m=0, \ldots, 5$ elements. In Table 1 we report the degrees of freedom $d o f_{C}$ of the non-fitting meshes and the degrees of freedom $d o f_{I}$ of the interface meshes together with the mesh size $h$. As we can see, the additional computational cost due to the interface mesh is negligible with respect to that of the global non-fitting mesh.

In the first simulation, we consider a linear interface with $\gamma(y)=0.4 y+$ 0.12 , and we test both a small coefficient jump with $\beta_{1}=0.1$ and $\beta_{2}=1$ and larger one with $\beta_{1}=10^{-2}$ and $\beta_{2}=10^{4}$. As an example, we plot in Figure 3 the analytical solution obtained with the first choice of coefficients.

In Table 2 we report the $L^{2}$-norm and the $H^{1}$-seminorm of the relative error $e_{h}$ and the corresponding estimated convergence rates $r_{0}$ and $r_{1}$. As we can see, the expected convergence rates are achieved by both the simulations, and the scheme is accurate even for considerably different coefficients.

In the second test we use the same choices of $\beta_{i} i=1,2$ as in the previous simulation, but now we consider the nonlinear interface with $\gamma(y)=0.3 y^{2}+$ $0.4 y-0.12$. In this case the analytical solution obtained with the first choice 


\begin{tabular}{|c|c|c|c|}
\hline$m$ & $d o f_{C}$ & $d o f_{I}$ & $h$ \\
\hline 0 & 16 & 10 & $7.22 \mathrm{e}-01$ \\
\hline 1 & 77 & 20 & $3.61 \mathrm{e}-01$ \\
\hline 2 & 337 & 40 & $1.81 \mathrm{e}-01$ \\
\hline 3 & 1409 & 82 & $9.03 \mathrm{e}-02$ \\
\hline 4 & 5761 & 166 & $4.51 \mathrm{e}-02$ \\
\hline 5 & 23297 & 334 & $2.26 \mathrm{e}-02$ \\
\hline
\end{tabular}

Table 1: Mesh parameters for unstructured grids with $46 \cdot 4^{m}$ elements. We report the degrees of freedom of the global mesh $\left(d o f_{C}\right)$ and of the interface mesh $\left(d o f_{I}\right)$ and the mesh size $h$.

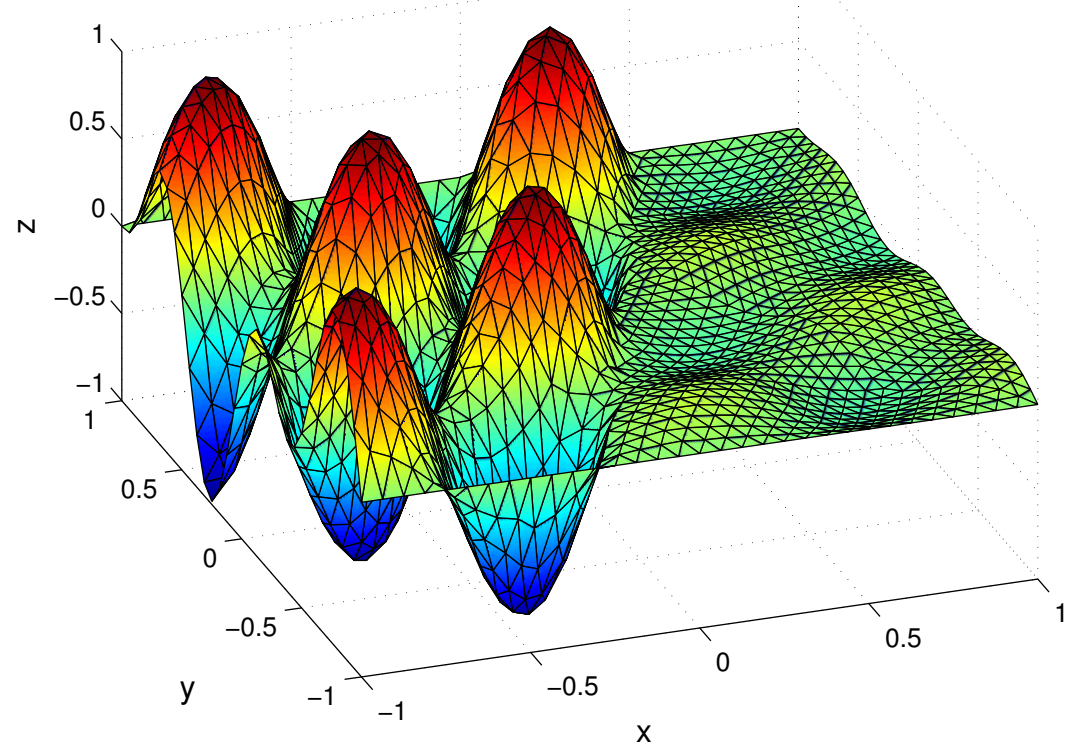

Figure 3: Analytical solution (33) of the problem (2) for $\beta_{1}=0.1$ and $\beta_{2}=1$ and a linear interface with $\gamma(y)=0.4 y+0.12$. 


\begin{tabular}{|c||c|c||c|c||c|c||c|c|}
\hline \multicolumn{1}{|c||}{} & \multicolumn{4}{c||}{$\beta_{1}=0.1, \beta_{2}=1$} & \multicolumn{4}{c|}{$\beta_{1}=10^{-2}, \beta_{2}=10^{4}$} \\
\hline$m$ & $\left\|e_{h}\right\|_{0}$ & $r_{0}$ & $\left|e_{h}\right|_{1}$ & $r_{1}$ & $\left\|e_{h}\right\|_{0}$ & $r_{0}$ & $\left|e_{h}\right|_{1}$ & $r_{1}$ \\
\hline 0 & $8.282-1$ & & $8.602 \mathrm{e}-1$ & & $8.269 \mathrm{e}-1$ & & $8.619 \mathrm{e}-1$ & \\
\hline 1 & $2.874 \mathrm{e}-1$ & 1.53 & $5.161 \mathrm{e}-1$ & 0.74 & $2.877 \mathrm{e}-1$ & 1.52 & $5.166 \mathrm{e}-1$ & 0.74 \\
\hline 2 & $7.910 \mathrm{e}-2$ & 1.86 & $2.738 \mathrm{e}-1$ & 0.91 & $7.908 \mathrm{e}-2$ & 1.86 & $2.738 \mathrm{e}-1$ & 0.92 \\
\hline 3 & $2.034 \mathrm{e}-2$ & 1.96 & $1.393 \mathrm{e}-1$ & 0.97 & $2.032 \mathrm{e}-2$ & 1.96 & $1.393 \mathrm{e}-1$ & 0.97 \\
\hline 4 & $5.137 \mathrm{e}-3$ & 1.99 & $7.014 \mathrm{e}-2$ & 0.99 & $5.133 \mathrm{e}-3$ & 1.99 & $7.013 \mathrm{e}-2$ & 0.99 \\
\hline 5 & $1.289 \mathrm{e}-3$ & 2.00 & $3.515 \mathrm{e}-2$ & 1.00 & $1.288 \mathrm{e}-3$ & 2.00 & $3.514 \mathrm{e}-2$ & 1.00 \\
\hline
\end{tabular}

Table 2: $L^{2}$-norm $\left\|e_{h}\right\|_{0}$ and $H^{1}$-seminorm $\left|e_{h}\right|_{1}$ of the relative errors and estimated convergence rates $r_{0}$ and $r_{1}$ for different values of the constants $\beta_{i}$ with $\gamma(y)=0.4 y+0.12$ on unstructured meshes.

of coefficients is plotted in Figure 4.

In Table 3 we report the $L^{2}$-norm and the $H^{1}$-seminorm of the relative error $e_{h}$ together with the estimated convergence rates $r_{0}$ and $r_{1}$. Also in this case, the expected convergence rates are achieved for both choices of $\beta_{i}$.

\subsection{Condition number}

Let us investigate now the behavior of the condition number of the matrices involved in the solution of (31) and of the Schur complement matrix (32). For this we consider a family of structured meshes and we set $\gamma(y)=c_{3}$. We choose $c_{3}$ suitably small so that for each mesh, the interface crosses only the elements included in the vertical strip $\Omega^{\Gamma}=\left[0,2 / 2^{i}\right] \times[-1,1], i=2, \ldots, 7$. All the following plots are in bi-logarithmic scale.

Let us first set $c_{3}=0.012$ and $\beta_{1}=0.1$ and $\beta_{2}=10^{n}$ for $n=0,2,4,6,8$. In Figure 5 we plot the condition numbers of $A, A_{I I}, A_{G G}, A_{S}$ versus $\beta_{2} / \beta_{1}$ 


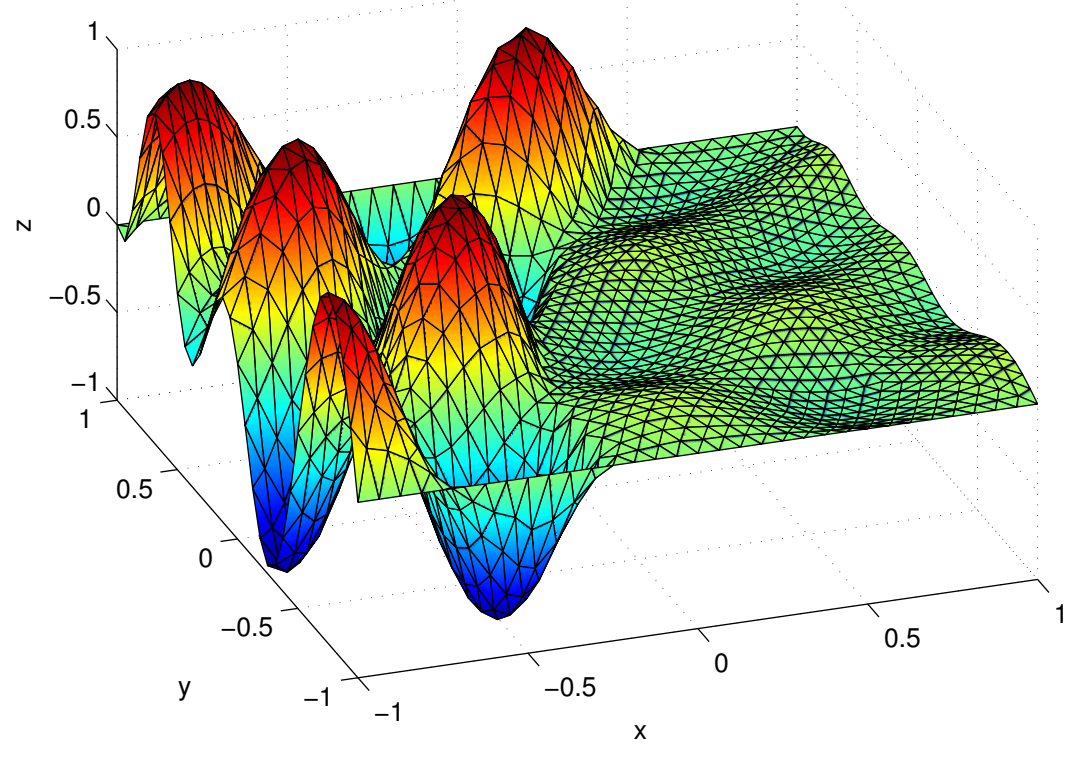

Figure 4: Analytical solution (33) of the problem (2) for $\beta_{1}=0.1$ and $\beta_{2}=1$ and a nonlinear interface with $\gamma(y)=0.3 y^{2}+0.4 y-0.12$. 


\begin{tabular}{|c||c|c||c|c||c|c||c|c|}
\hline \multicolumn{1}{|c||}{ Mesh } & \multicolumn{4}{c||}{$\beta_{1}=0.1, \beta_{2}=1$} & \multicolumn{4}{c|}{$\beta_{1}=10^{-2}, \beta_{2}=10^{4}$} \\
\hline$m$ & $\left\|e_{h}\right\|_{0}$ & $r_{0}$ & $\left|e_{h}\right|_{1}$ & $r_{1}$ & $\left\|e_{h}\right\|_{0}$ & $r_{0}$ & $\left|e_{h}\right|_{1}$ & $r_{1}$ \\
\hline 0 & $1.040 \mathrm{e} 0$ & & $1.048 \mathrm{e} 0$ & & $1.076 \mathrm{e} 0$ & & $1.053 \mathrm{e} 0$ & \\
\hline 1 & $4.185 \mathrm{e}-1$ & 1.31 & $6.088 \mathrm{e}-1$ & 0.78 & $4.225 \mathrm{e}-1$ & 1.35 & $6.105 \mathrm{e}-1$ & 0.79 \\
\hline 2 & $1.189 \mathrm{e}-1$ & 1.82 & $3.137 \mathrm{e}-1$ & 0.96 & $1.197 \mathrm{e}-1$ & 1.82 & $3.143 \mathrm{e}-1$ & 0.96 \\
\hline 3 & $3.072 \mathrm{e}-2$ & 1.95 & $1.591 \mathrm{e}-1$ & 0.98 & $3.089 \mathrm{e}-2$ & 1.95 & $1.593 \mathrm{e}-1$ & 0.98 \\
\hline 4 & $7.761 \mathrm{e}-3$ & 1.98 & $7.999 \mathrm{e}-2$ & 0.99 & $7.800 \mathrm{e}-3$ & 1.99 & $8.009 \mathrm{e}-2$ & 0.99 \\
\hline 5 & $1.947 \mathrm{e}-3$ & 1.99 & $4.009 \mathrm{e}-2$ & 1.00 & $1.957 \mathrm{e}-3$ & 1.99 & $4.014 \mathrm{e}-2$ & 1.00 \\
\hline
\end{tabular}

Table 3: $L^{2}$-norm $\left\|e_{h}\right\|_{0}$ and $H^{1}$-seminorm $\left|e_{h}\right|_{1}$ of the relative errors and estimated convergence rates $r_{0}$ and $r_{1}$ for different values of the constants $\beta_{i}$ with $\gamma(y)=0.3 y^{2}+0.4 y-0.12$ on several unstructured meshes.

and we see that they are all proportional to $\beta_{2} / \beta_{1}$. We performed other simulations in which we changed both the values of $\beta_{i}$, and we saw that the condition numbers actually depend only on the coefficients ratio. The previous result can be summarized as follows (the symbol $\propto$ means proportional to)

$$
K(A) \propto \frac{\beta_{2}}{\beta_{1}}, \quad K\left(A_{G G}\right) \propto \frac{\beta_{2}}{\beta_{1}}, \quad K\left(A_{I I}\right) \propto \frac{\beta_{2}}{\beta_{1}}, \quad K\left(A_{S}\right) \propto \frac{\beta_{2}}{\beta_{1}} .
$$

Then in Figure 6 we plot the condition numbers versus the mesh size $h$. We find that, as expected, $K\left(A_{G G}\right)$ and $K\left(A_{S}\right)$ are proportional to $h^{-2}$, while $K(A)$ is proportional to $h^{-1}$ and $K\left(A_{I I}\right)$ is proportional to $h$, obtaining

$$
K(A) \propto \frac{1}{h}, \quad K\left(A_{G G}\right) \propto \frac{1}{h^{2}}, \quad K\left(A_{I I}\right) \propto h, \quad K\left(A_{S}\right) \propto \frac{1}{h^{2}} .
$$

Now we study the behavior of the condition numbers when the interface gets closer to $x=0$, in order to consider increasingly anisotropic interface 
elements. We set $\beta_{1}=0.1$ and $\beta_{2}=1$ and we take $\gamma(y)=c_{3}=0.012 \cdot 10^{-n}$ with $n=0,2,4,6,8$. In Figure 7 we plot only the behavior of the condition numbers of $A$ and $A_{I I}$ with respect to different positions of the interface for different mesh sizes $h$, since we have verified that the condition numbers of $A_{G G}$ and $A_{S}$ are not affected by the position of the interface. As we can see, $K(A)$ and $K\left(A_{I I}\right)$ are both proportional to $c_{3}^{-1}$. The condition number of $A_{I I}$ is larger on coarser grids, because in this case the interface elements are very anisotropic. As the grid is refined, the interface elements become more and more isotropic and the condition number decreases. We remark that, for each choice of the position of the interface, $c_{3}$ is actually the minimum of the edges of the interface mesh. Hence we can state the following relations

$$
K(A) \propto \frac{1}{c_{3}} \quad K\left(A_{G G}\right) \propto 1, \quad K\left(A_{I I}\right) \propto \frac{1}{c_{3}} \quad K\left(A_{S}\right) \propto 1 .
$$

In Figure 8 we report the behavior of the condition numbers versus the mesh size $h$, for different values of the interface position. We remark that our experiments show that the error is not affected by the presence of anisotropic elements.

We can summarize the results reported in (34), (35) and (36) by the following relations

$$
\begin{aligned}
& K(A) \propto \frac{\beta_{2}}{\beta_{1} h c_{3}}, \quad K\left(A_{G G}\right) \propto \frac{\beta_{2}}{\beta_{1} h^{2}}, \\
& K\left(A_{I I}\right) \propto \frac{\beta_{2} h}{\beta_{1} c_{3}}, \quad K\left(A_{S}\right) \propto \frac{\beta_{2}}{\beta_{1} h^{2}} .
\end{aligned}
$$

These relations have been checked performing several simulations on different meshes, with different interface positions and coefficient ratios $\beta_{2} / \beta_{1}$ and fitting, in the least-squares sense, the logarithm of the condition numbers 


\begin{tabular}{|c|c|c|c|}
\hline & $\alpha_{r}$ & $\alpha_{h}$ & $\alpha_{\gamma}$ \\
\hline$K(A)$ & 0.995 & -1.210 & -0.970 \\
\hline$K\left(A_{G G}\right)$ & 0.994 & -2.006 & 0.003 \\
\hline$K\left(A_{I I}\right)$ & 0.999 & 0.881 & -0.988 \\
\hline$K\left(A_{S}\right)$ & 0.995 & -2.004 & 0.004 \\
\hline
\end{tabular}

Table 4: Least-squares approximation of exponents of (38) for the condition numbers $K(\cdot)$. The values agree with (37).

with respect to the logarithm of the following function

$$
\left(\frac{\beta_{2}}{\beta_{1}}\right)^{\alpha_{r}} h^{\alpha_{h}} c_{3}^{\alpha_{\gamma}} .
$$

The values of $\alpha_{r}, \alpha_{h}, \alpha_{\gamma}$ collected in Table 4 are in good agreement with (37). In particular, we observe that the condition number of $A$ and $A_{I I}$ increases when the interface gets closer to element edges ( $c_{3}$ tends to zero). In this case one could try to use a different way for the construction of the local sub-grid avoiding elements with one edge too small with respect to the others.

\subsection{Comparison to immersed interface method}

In the last set of experiments, we compare our method with the immersed interface method (IIM) introduced in $[15,16,33]$. The IIM is based on the construction of suitable basis functions on the elements crossed by the interface which satisfy the transmission conditions of problem (2). Notice that the resulting numerical solution can present jumps along the interelement boundaries. We consider Problem (2) with solution given by (33), and compute the $L^{2}$ relative errors obtained on the family of unstructured meshes with 
the nonlinear interface used in the previous simulations. We take $\beta_{1}=0.1$ and different increasing values of the coefficient $\beta_{2}=10^{n}$ for $n=0,4,8$. The results reported in Figure 9 show that the relative errors of our method do not change significantly when we increase coefficient $\beta_{2}$, unlike the errors produced by IIM, which behaves more irregularly as $\beta_{2}$ increases, especially on the coarser grids.

In the last test, we consider the two simulations reported in [33, page 182]. We set again $\Omega=[-1,1]^{2}$, while the interface is the circle centered at the origin with radius $r_{0}$. Following [33], we set $r_{0}=\pi / 6.28$ and choose $f$ and the boundary conditions so that the exact solution is

$$
u(x, y)= \begin{cases}\frac{\left(x^{2}+y^{2}\right)^{3 / 2}}{\beta_{1}} & \text { if } x^{2}+y^{2} \leq r_{0}^{2} \\ \frac{\left(x^{2}+y^{2}\right)^{3 / 2}}{\beta_{2}}+\left(\frac{1}{\beta_{1}}-\frac{1}{\beta_{2}}\right) r_{0}^{3} & \text { otherwise }\end{cases}
$$

In Figure 10 we report the $L^{2}$-norm and $H^{1}$-seminorm of the relative errors for test 1 , obtained setting $\beta_{1}=1$ and $\beta_{2}=1000$, and for test 2 , in which we set $\beta_{1}=1000$ and $\beta_{2}=1$. The results are comparable with those reported in [33] where the error is evaluated in $L^{\infty}$-norm. Also in this case the expected convergence rates are achieved by our method.

\section{Conclusions}

In this work we introduced a new numerical method for the approximation of elliptic interface problems. It is based on a local enrichment of the global finite element space, so that the global mesh needs not to be modified. The local enrichment is achieved using shape functions of the same type as those of the global finite element space, so that the approximation is 
conforming. In this work we studied a correction based on piecewise-linear polynomials, but the approach can be generalized to higher order approximations. We performed error analysis and we proved that the approach allows to recover the optimal rates of convergence in $L^{2}$ and in $H^{1}$, taking advantage of the local regularity of the solution. Several numerical simulations confirmed the theoretical results. Since our method does not require a modification of the global mesh, it turns out to be particularly attractive for time dependent problems, in which the interface is not static. We plan to investigate the application of this new approach to parabolic problems with moving interfaces.

\section{References}

[1] F. B. Jensen, W. A. Kuperman, M. B. Porter, H. Schmidt, Computational Ocean Acoustics, Springer-Verlag New York Inc., 2011.

[2] F. Muir, J. Dellinger, J. Etgen, D. Nichols, Modeling elastic fields across irregular boundaries, Geophysics 57 (1992) 189-193.

[3] A. Yefet, E. Turkel, Fourth order compact implicit method for the Maxwell equations with discontinuous coefficients, Appl. Numer. Math. 33 (2000) 125-134.

[4] K. H. Karlsen, N. H. Risebro, J. D. Towers, Upwind difference approximations for degenerate parabolic convection-diffusion equations with a discontinuous coefficient, IMA J. Numer. Anal. 22 (2002) 623-664.

[5] H.-G. Kim, A new coupling strategy for fluid-solid interaction problems 
by using the interface element method, Internat. J. Numer. Methods Engrg. 81 (2010) 403-428.

[6] X. Feng, Fully discrete finite element approximations of the NavierStokes-Cahn-Hilliard diffuse interface model for two-phase fluid flows, SIAM J. Numer. Anal. 44 (2006) 1049-1072 (electronic).

[7] C. S. Peskin, The immersed boundary method, Acta Numer. 11 (2002) $479-517$.

[8] D. Boffi, L. Gastaldi, L. Heltai, C. S. Peskin, On the hyper-elastic formulation of the immersed boundary method, Comput. Methods Appl. Mech. Engrg. 197 (2008) 2210-2231.

[9] A. Ibrahimbegović, D. Markovič, Strong coupling methods in multiphase and multi-scale modeling of inelastic behavior of heterogeneous structures, Computer Methods in Applied Mechanics and Engineering 192 (2003) 3089-3107.

[10] M. Hautefeuille, J.-B. Colliat, A. Ibrahimbegović, H. G. Matthies, P. Villon, A multi-scale approach to model localized failure with softening, Computers and Structures 94-95 (2012) 83-95.

[11] I. Babuška, The finite element method for elliptic equations with discontinuous coefficients, Computing (Arch. Elektron. Rechnen) 5 (1970) $207-213$.

[12] J. H. Bramble, J. T. King, A finite element method for interface problems in domains with smooth boundaries and interfaces, Adv. Comput. Math. 6 (1996) 109-138 (1997). 
[13] J. Xu, Estimate of the convergence rate of the finite element solutions to elliptic equation of second order with discontinuous coefficients, Natural Science Journal of Xiangtan University 1 (1982) 1-5.

[14] Z. Chen, J. Zou, Finite element methods and their convergence for elliptic and parabolic interface problems, Numer. Math. 79 (1998) 175202.

[15] R. J. LeVeque, Z. L. Li, The immersed interface method for elliptic equations with discontinuous coefficients and singular sources, SIAM J. Numer. Anal. 31 (1994) 1019-1044.

[16] Z. Li, The immersed interface method using a finite element formulation, Appl. Numer. Math. 27 (1998) 253-267.

[17] I. Babuška, J. M. Melenk, The partition of unity method, Internat. J. Numer. Methods Engrg. 40 (1997) 727-758.

[18] T. Belytschko, N. Moës, S. Usui, C. Parimi, Arbitrary discontinuities in finite elements, International Journal for Numerical Methods in Engineering 50 (2001) 993-1013.

[19] A. Hansbo, P. Hansbo, An unfitted finite element method, based on Nitsche's method, for elliptic interface problems, Comput. Methods Appl. Mech. Engrg. 191 (2002) 5537-5552.

[20] R. Glowinski, Y. Kuznetsov, On the solution of the Dirichlet problem for linear elliptic operators by a distributed Lagrange multiplier method, C. R. Acad. Sci. Paris Sér. I Math. 327 (1998) 693-698. 
[21] R. Glowinski, Y. Kuznetsov, Distributed Lagrange multipliers based on fictitious domain method for second order elliptic problems, Comput. Methods Appl. Mech. Engrg. 196 (2007) 1498-1506.

[22] F. Ilinca, J.-F. Hétu, A finite element immersed boundary method for fluid flow around rigid objects, International Journal for Numerical Methods in Fluids 65 (2011) 856-875.

[23] F. Ilinca, J.-F. Hétu, A finite element immersed boundary method for fluid flow around moving objects, Computers and Fluids 39 (2010) 1656-1671.

[24] K. Lemrabet, Régularité de la solution d'un problème de transmission, J. Math. Pures Appl. (9) 56 (1977) 1-38.

[25] R. B. Kellogg, Singularities in interface problems, in: Numerical Solution of Partial Differential Equations, II (SYNSPADE 1970) (Proc. Sympos., Univ. of Maryland, College Park, Md., 1970), Academic Press, New York, 1971, pp. 351-400.

[26] S. Nicaise, Polygonal interface problems, volume 39 of Methoden und Verfahren der Mathematischen Physik [Methods and Procedures in Mathematical Physics], Verlag Peter D. Lang, Frankfurt am Main, 1993.

[27] A. Ženíšek, The finite element method for nonlinear elliptic equations with discontinuous coefficients, Numer. Math. 58 (1990) 51-77.

[28] M. Feistauer, A. Ženíšek, Finite element solution of nonlinear elliptic problems, Numer. Math. 50 (1987) 451-475. 
[29] R. A. Adams, J. J. F. Fournier, Sobolev spaces, volume 140 of Pure and Applied Mathematics (Amsterdam), Elsevier/Academic Press, Amsterdam, second edition, 2003.

[30] S. C. Brenner, L. R. Scott, The mathematical theory of finite element methods, volume 15 of Texts in Applied Mathematics, Springer, New York, third edition, 2008.

[31] E. M. Stein, Singular integrals and differentiability properties of functions, Princeton Mathematical Series, No. 30, Princeton University Press, Princeton, N.J., 1970.

[32] W. McLean, Strongly elliptic systems and boundary integral equations, Cambridge University Press, Cambridge, 2000.

[33] Z. Li, K. I., The immersed interface method, volume 33 of Frontiers in Applied Mathematics, Society for Industrial and Applied Mathematics (SIAM), Philadelphia, PA, 2006. Numerical solutions of PDEs involving interfaces and irregular domains. 

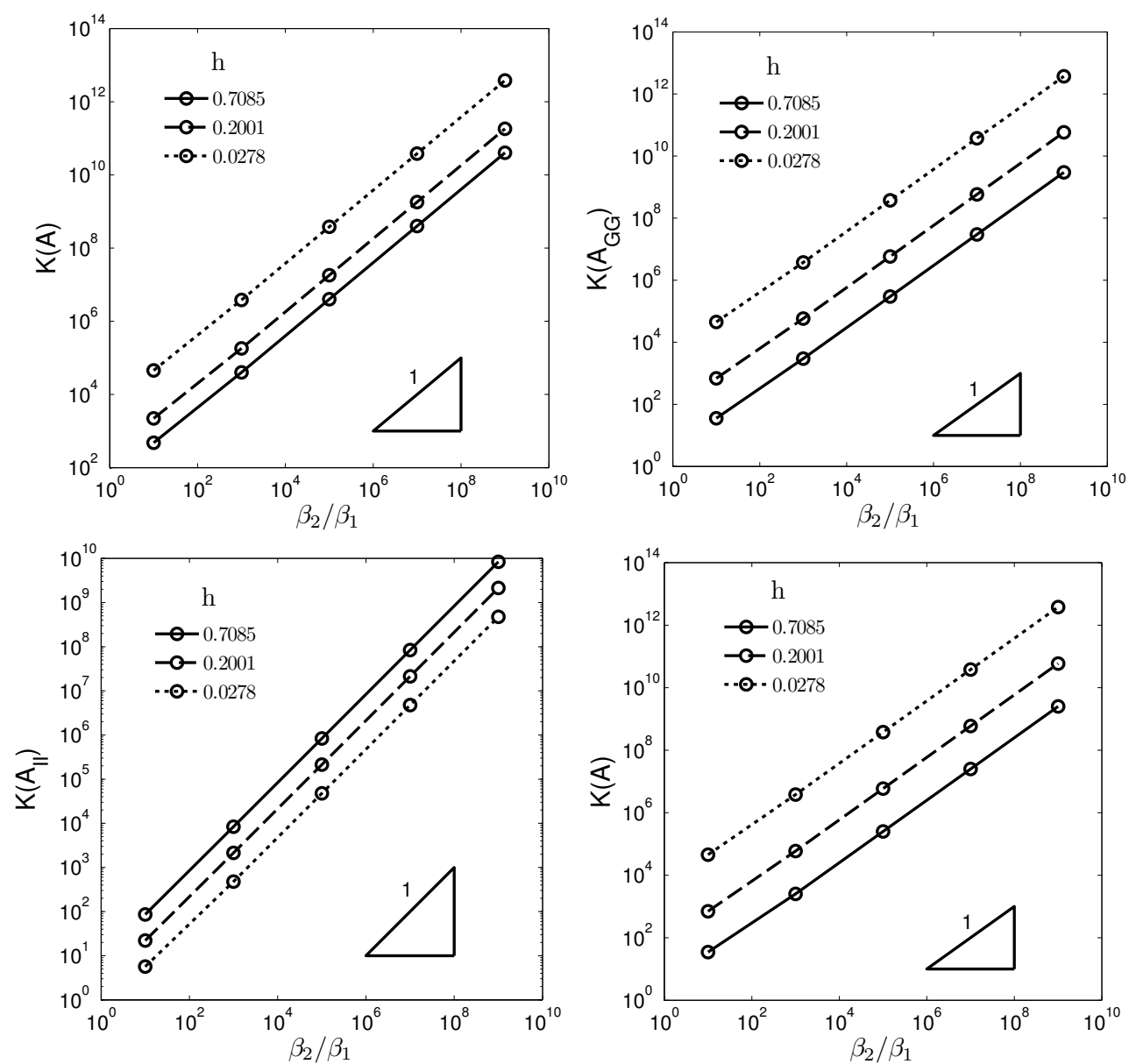

Figure 5: Condition numbers of the matrices $A, A_{G G}, A_{I I}, A_{S}$ versus the ratio $\beta_{2} / \beta_{1}$ for the test problem with $\beta_{1}=0.1$ and several different values of $\beta_{2}$. 

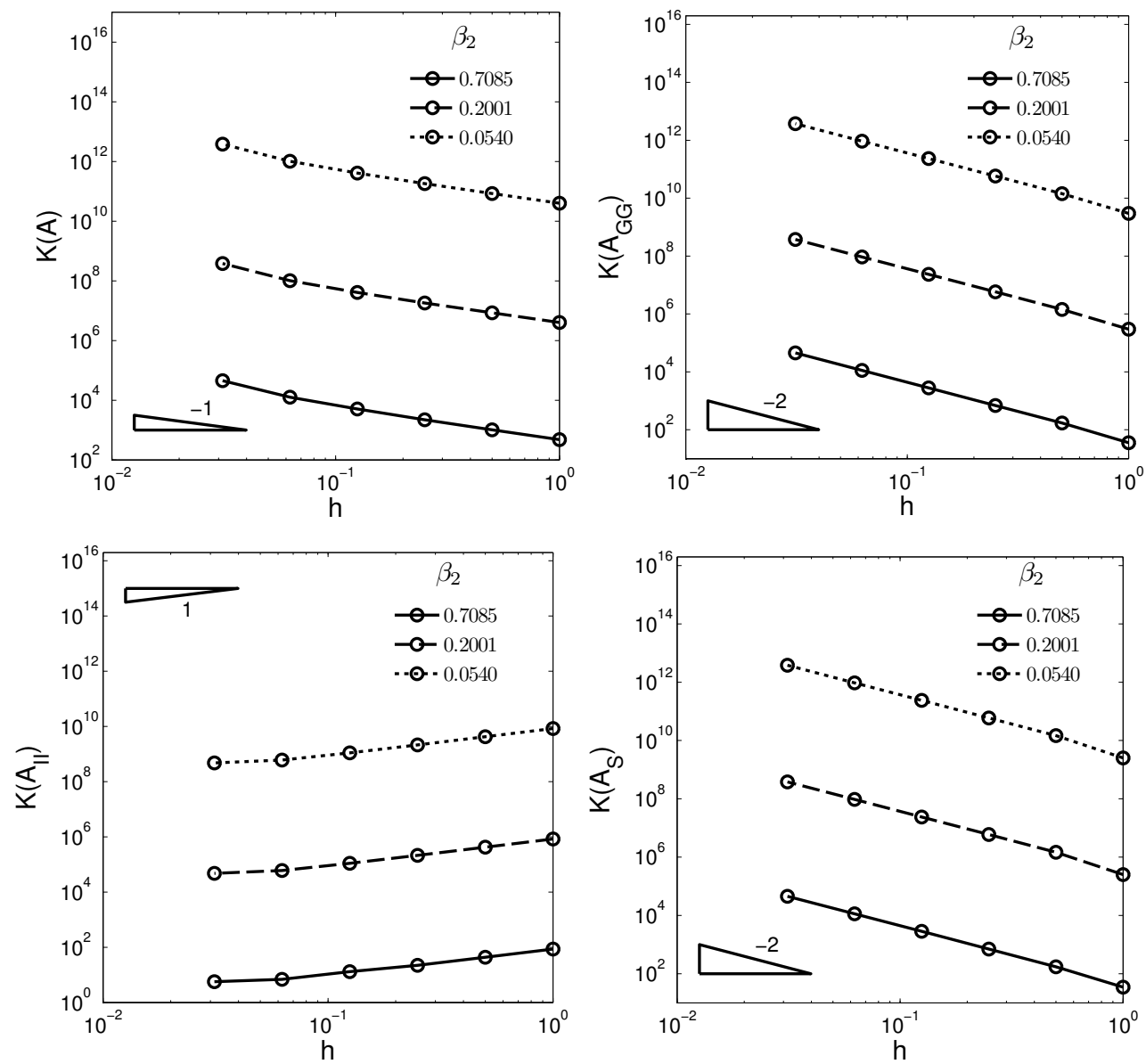

Figure 6: Condition numbers of the matrices $A, A_{G G}, A_{I I}, A_{S}$ with respect to the mesh size $h$ for the problem with solution (33) with $\beta_{1}=0.1$ and several different values of $\beta_{2}$. 

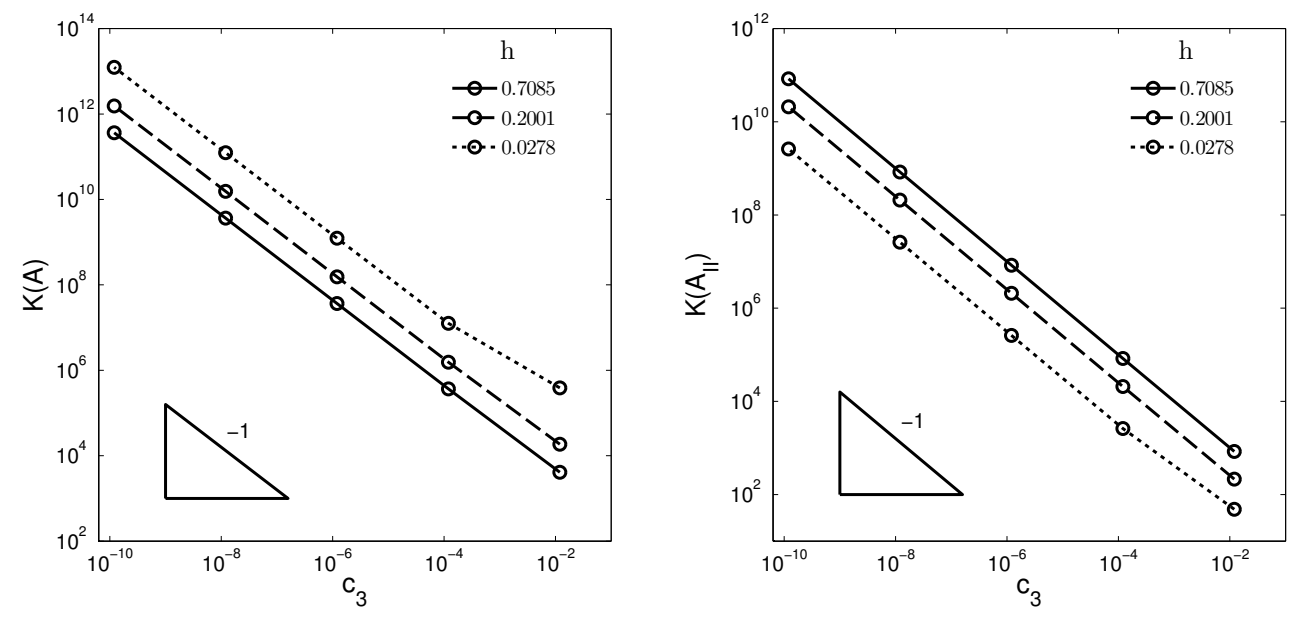

Figure 7: Condition numbers of the matrices $A, A_{I I}$ versus the interface position $c_{3}$ for the problem with solution (33) with $\beta_{1}=0.1$ and $\beta=1$ on several different meshes with size $h$.
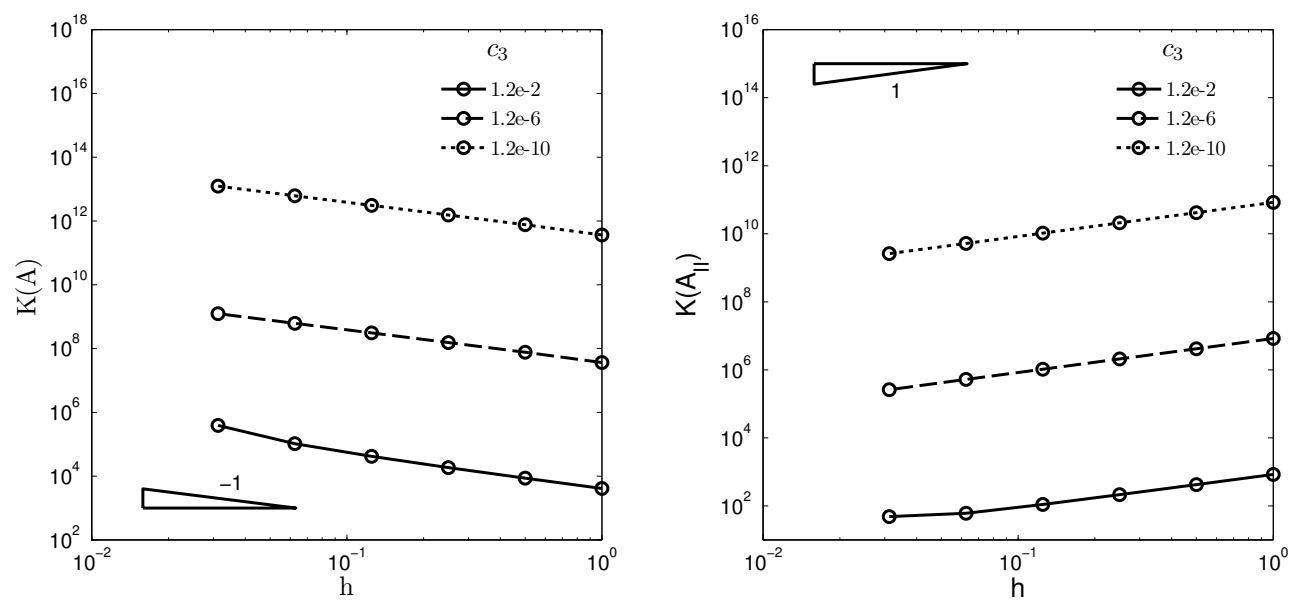

Figure 8: Condition numbers of the matrices $A, A_{I I}$ versus $h$ for the test problem with $\beta_{1}=0.1$ and $\beta=1$ and several different values of $c_{3}$, which determine the position of the interface. 


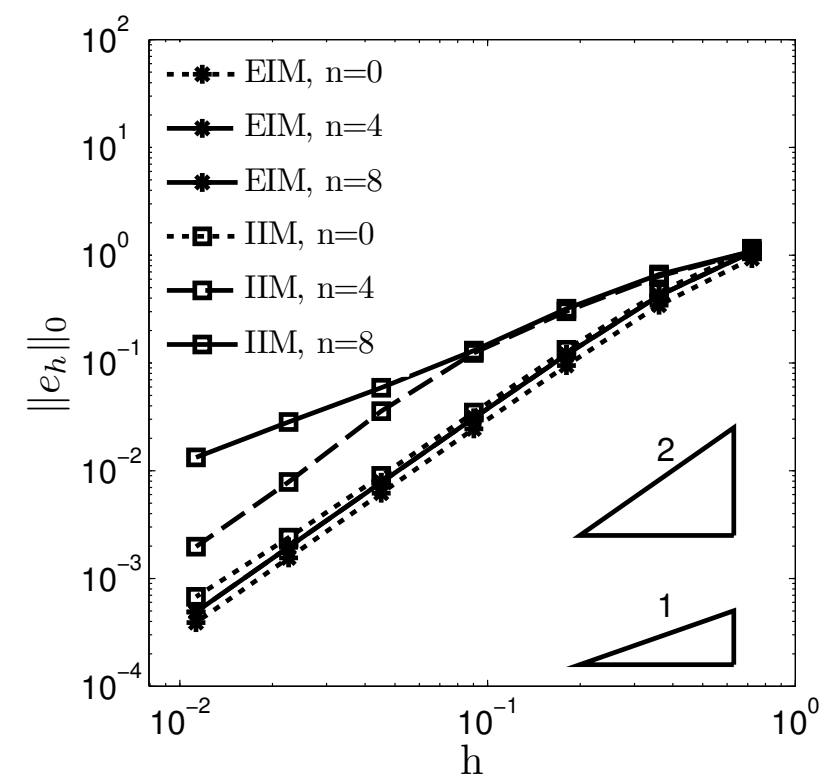

Figure 9: Comparison of the errors for the test problem for increasing values of $\beta_{2}=$ $10^{n}, n=0,4,8$. The lines with a square mark show the errors of the enriched interface method (EIM) described in this paper while the lines with a star mark are those obtained with the immersed interface method (IIM). 


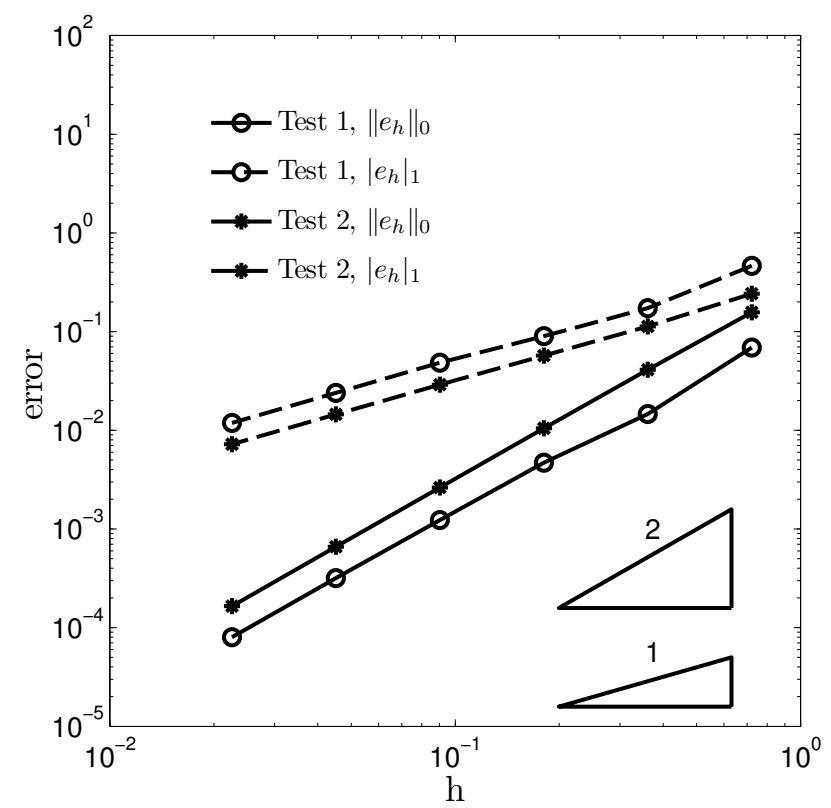

Figure 10: $L^{2}$-norm $\left\|e_{h}\right\|_{0}$ and $H^{1}$-seminorm $\left|e_{h}\right|_{1}$ of the relative errors for test problem (39). 